\title{
Effect of the cross-linker on the general performance and temperature dependent behaviour of a molecularly imprinted polymer catalyst of a Diels-Alder reaction
}

Henning Henschel $^{\mathrm{a}}$, Nicole Kirsch ${ }^{\mathrm{a}}$, Jimmy Hedin-Dahlström ${ }^{\mathrm{a}}$, Michael J. Whitcombe ${ }^{\mathrm{b}}$, Susanne Wikman ${ }^{\mathrm{a}}$, Ian A. Nicholls $\mathrm{s}^{\mathrm{a}, \mathrm{c}, *}$

${ }^{a}$ Bioorganic \& Biophysical Chemistry Laboratory, School of Natural Sciences, Linnoeus University, SE-391 82 Kalmar, Sweden.

${ }^{b}$ Cranfield Health, Cranfield University, Cranfield, Bedfordshire, MK43 0AL, U.K.

${ }^{c}$ Department of Biochemistry \& Organic Chemistry, Uppsala University, SE-751 23 Uppsala, Sweden.

Author to whom correspondence should be addressed:

Ian A. Nicholls: Tel: +46 480446258 Fax: +46 480446244

E-mail: ian.nicholls@lnu.se 


\begin{abstract}
Here we present a series of molecularly imprinted polymers capable of catalysing the DielsAlder reaction between benzyl 1,3-butadienylcarbamate (1) and $N, N$-dimethyl acrylamide (2). The polymer systems studied here demonstrated an unusual cross-linker and temperature dependent behaviour, namely that polymer catalysis of the Diels-Alder reaction was lower at elevated temperature, in contrast to the solution reaction. Furthermore, not only was the catalytic activity significantly influenced by the choice of cross-linker, but in a similar fashion also the extent of the temperature effect, indicating a close relationship between catalysis and the observed inhibition. Molecular dynamics simulations of both the polymer systems studied were used to provide insight into the molecular background of transition state stabilisation, and differences in properties of the systems based on different cross-linkers.
\end{abstract}

\title{
Graphical Abstract
}

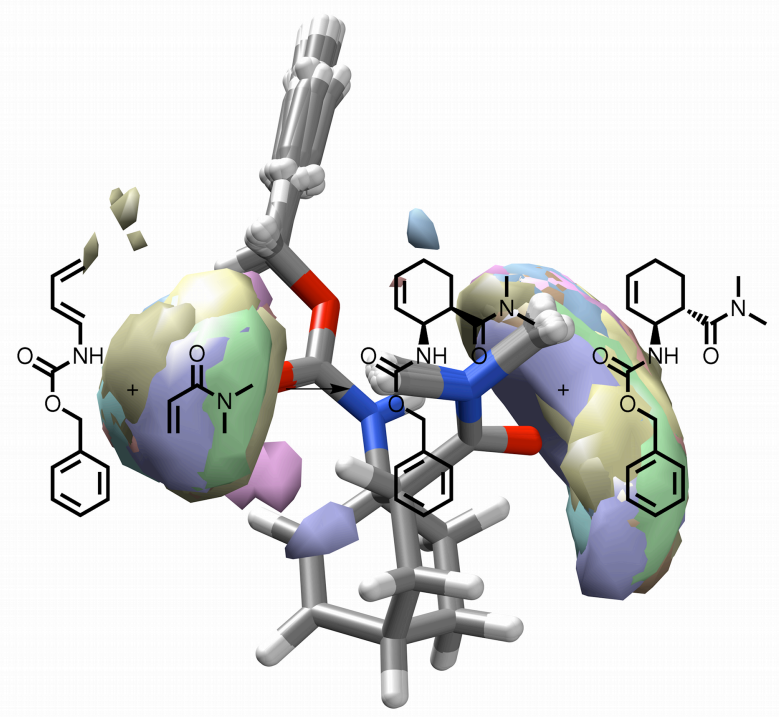

Highlights:

- Recently described effect of high temperature on a MIP observed for another system.

- Different cross-linker allows for more detailed study of effect.

- MD studies give background to differences between polymer systems. 
Keywords: Diels-Alder reaction, molecularly imprinted polymer, molecular dynamics simulation, plastic antibody, transition state analogue

Abbreviations: ACHN, azobis(cyclohexanecarbonitrile); BHT, 3,5-di-tert-butyl-4-hydroxytoluene; DVB, divinylbenzene; EGMA, ethylene glycol dimethacrylate, MAA, methacrylic acid; MD, molecular dynamics; TSA, transition state analogue. 


\section{Introduction}

The Diels-Alder reaction is one of the most powerful synthetic tools for the creation of carbon-carbon bonds. It is widely used in the preparation of physiologically active compounds.[1] This makes it an important target for the development of catalytic systems.[2-4] One approach towards the rational design of catalysts is the production of antibodies against a transition state analogue (TSA) as demonstrated for the Diels-Alder reaction by the Janda group.[5,6]

An approach analogous to the production of catalytic antibodies can be seen in the preparation of catalytic molecularly imprinted polymers. Molecular imprinting is carried out by polymerisation of commonly two types of monomer (one functional monomer and one crosslinker) in presence of a template, giving, after removal of the template, a polymer with selective recognition sites for the template - essentially a plastic antibody.[7-14] If a structure similar to a certain transition state is used as template, the resulting polymer can - just as a catalytic antibody - be expected to stabilise the transition state, catalysing the corresponding reaction.[13] While a growing number of catalytic polymers have been designed and synthesised for a number of different reaction types, e.g. ester hydrolysis[15-25], transamination[26], $\beta$-elimination[27,28], only a few reports deal with C-C bond formation, e.g. aldol[29,30], Suzuki[31], and Diels-Alder reactions[32-34].

We recently described a previously unreported temperature dependent behaviour of catalytic molecularly imprinted polymers,[35] where a polymer system, based on methacrylic acid (MAA) as functional monomer and divinylbenzene (DVB) as cross-linker, was designed to catalyse the Diels-Alder reaction shown in Figure 1. The template used in this study was adapted from the transition state analogue developed by Gouverneur et al.[5] in the production of catalytic antibodies for this [4+2] cycloaddition. While successfully enhancing the rate of the reaction up to 20 -fold compared to the solution reaction at room temperature, it 
was noted, however, that at elevated temperature the polymers were shown to lower the rate of the reaction instead. Here further insights into this phenomenon have been obtained through a combination of studies using polymers synthesised using an alternative crosslinking agent, and including one additional temperature step in the evaluation, while keeping the same functional monomer and template, granting direct comparability to the results presented previously. Moreover, a series of molecular dynamics studies was conducted, providing insight into which interactions are of importance for template, as well as rationalizing the effect of the change in cross-linker.<smiles>C=C/C=C\NC(=O)OCc1ccccc1</smiles>

Figure 1. The Diels-Alder reaction studied in ref [35] and this article.

\section{Materials and Methods}

\section{General.}

Reagents were purchased from Aldrich (Germany). Inhibitors from ethylene glycol dimethacrylate (EGMA) were removed by extraction with $0.1 \mathrm{M}$ sodium hydroxide solution. The EGMA was subsequently dried over magnesium sulfate and stored at $4{ }^{\circ} \mathrm{C}$. Treatment of other substances prior to their use is described in ref [35].

\section{Synthesis of Diels-Alder products and TSAs}

The endo- and exo-transition state analogues (5 and 6) were synthesized in six steps with an overall yield of 35 and $11 \%$, respectively, as summarized in Figure 2. A detailed description of the synthesis can be found in ref [35]. 


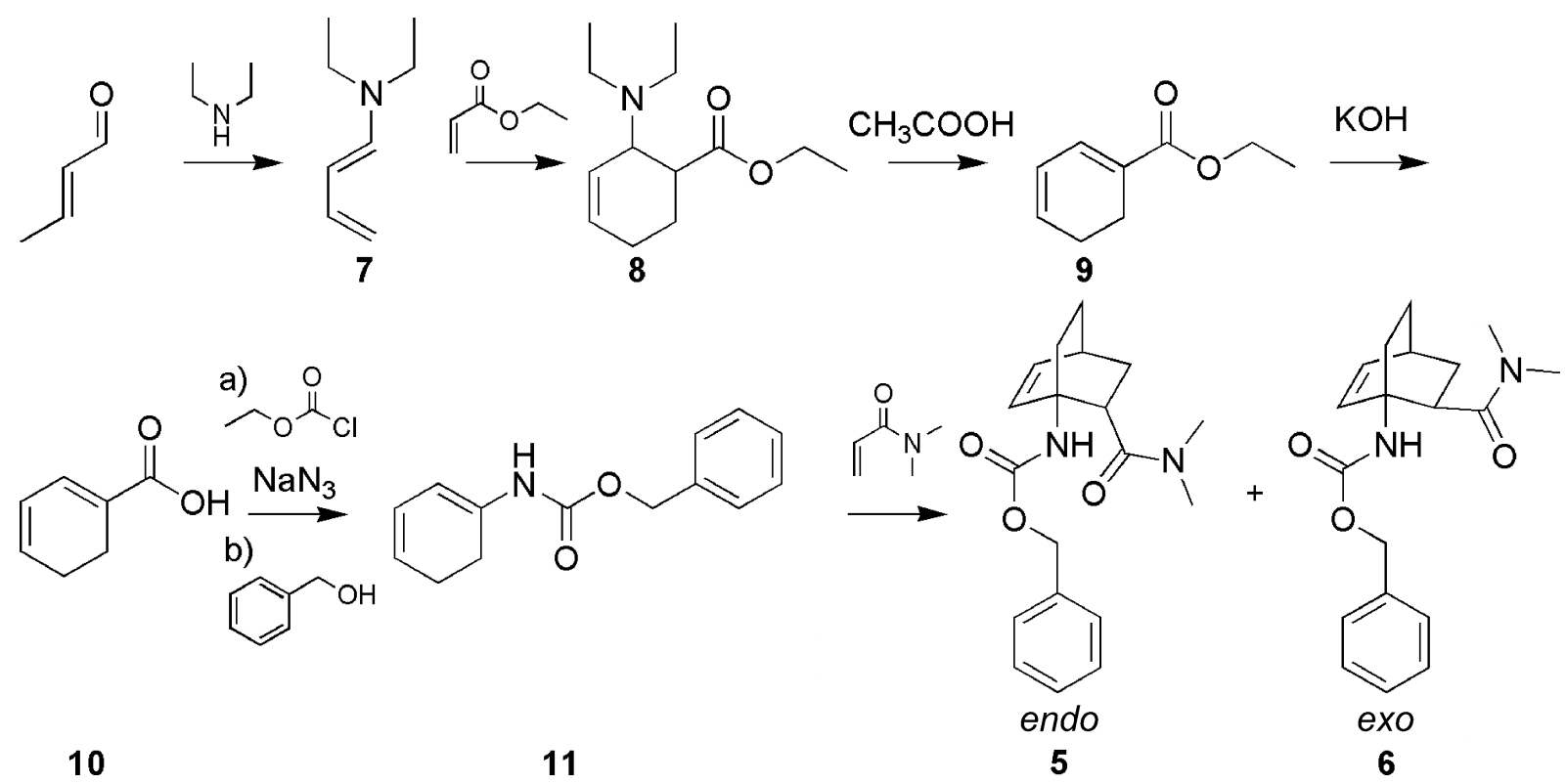

Figure 2. Synthesis of the endo- (5) and exo- (6) transition state analogues (TSAs).

Polymer synthesis.

Polymers were prepared, as previously described for DVB-based polymers,[35] using $0.0933 \mathrm{~g}(0.284 \mathrm{mmol}, 5 \mathrm{~mol} \%)$ TSA (5/6), $0.0979 \mathrm{~g}$ (1.14 mmol; $20 \mathrm{~mol} \%)$ MAA, and $0.921 \mathrm{~g}$ (4.55 mmol, $80 \mathrm{~mol} \%)$ EGMA for the synthesis of $1 \mathrm{~g}$ polymer. Polymerization was performed in $2 \mathrm{~mL}$ toluene, with $\mathrm{ACHN}$ ( $1 \mathrm{~mol} \%$ relative to double bonds) as initiator. To remove the template and additional fines, Soxhlet extraction was carried out in acetone. More complete template removal from the polymer was achieved by packing the polymer into a stainless steel HPLC column and subjecting it to extensive washing under a variety of conditions, as described elsewhere.[36] Control polymers were prepared in the same manner but in the absence of template.

\section{Batch binding studies.}

A solution of 5 or 6 in toluene $(0.5 \mathrm{~mL} ; 0.1$ and $1 \mathrm{mM}$, respectively) was shaken with $5 \pm 0.1 \mathrm{mg}$ polymer overnight. The polymer was filtered off and the concentration of the TSA remaining in solution determined by HPLC performed on a HP1050 or a HP1090 system using a Beckman silica column $(250 \mathrm{~mm} \times 4.6 \mathrm{~mm}$ i.d. $)$. Polymer suspensions were filtered 
through $2 \mu \mathrm{m}$ PTFE membrane filter cartridges prior to HPLC analysis. Elution was carried out in $90 \%$ heptane containing $0.1 \%$ acetic acid and $10 \%$ isopropanol at a flow rate of 1 $\mathrm{mL} / \mathrm{min}$ with UV detection at $220 \mathrm{~nm}$.

\section{Swelling studies.}

Polymer $(1 \mathrm{~mL})$ was weighed into a $5 \mathrm{~mL}$ glass cylinder, and $3 \mathrm{~mL}$ of toluene were added. The cylinder was sealed with a glass stopper and left standing for $12 \mathrm{~h}$. The increase in polymer volume (swelling, $\mathrm{SW}$ ), was recorded and the specific swelling $\left(\mathrm{SW}_{\mathrm{sp}}\right)$ calculated according to equation 1.

$S W_{s p}=\frac{S W}{m}$

\section{Kinetics studies.}

A solution was prepared containing benzyl 1,3-butadienylcarbamate (1, $20 \mathrm{mM})$, $N, N$-dimethyl acrylamide $(20 \mathrm{mM})$ and BHT $(5 \mathrm{mg} / 10 \mathrm{~mL}$ solution) in toluene. Portions $(3 \mathrm{~mL})$ of this solution were added to pressure tubes containing $100 \mathrm{mg}$ polymer (no polymer in case of the solvent reaction). The tubes were purged with nitrogen and heated to the respective temperature $\left(120,40\right.$, and $\left.20{ }^{\circ} \mathrm{C}\right)$ using an oil bath. At appropriate intervals reaction was stalled by cooling on ice and samples of $1 \mathrm{~mL}$ were taken. The samples were centrifuged for $5 \mathrm{~min}\left(10000 \mathrm{rcf}, 20^{\circ} \mathrm{C}\right)$, the supernatant filtered, and HPLC analyses performed in duplicate to determine the amount of product formed. Elution was carried out with heptane containing $0.1 \%$ acetic acid and $15 \%$ isopropanol at a flow rate of $1 \mathrm{~mL} / \mathrm{min}$ with UV detection at $210 \mathrm{~nm}$. Afterwards, the polymer was re-suspended, the mixture returned to the pressure tube, the tube was purged with nitrogen, sealed and heating was resumed. Studies using inhibitor were performed accordingly.

\footnotetext{
${ }^{1}$ In ref. [29] the corresponding formula is wrongly written with density $d$ instead of mass $m$. Results, however, are correct.
} 
All calculations were performed using the AMBER10[37] program package. Studies of the prepolymerization mixture were set up and conducted as has been described by Karlsson et al.[38]. Models of the respective prepolymerization mixtures were set up containing 20 molecules of the respective TSA, 80 molecules of MAA, 320 molecules representing the cross-linker (divinylbenzene was modelled as a 1:1 mixture between para- and meta-isomer), 1320 toluene molecules and 4 molecules of ACHN. Simulations were run for $20 \mathrm{~ns}$ at $310 \mathrm{~K}$ using a $9.0 \AA$ cut-off for non-bonded interactions. 


\section{Results}

The subject of this study is the influence of an imprinted polymer system with EGMA as cross-linker at different temperatures on the Diels-Alder reaction shown in Figure 1. This reaction has been the subject of a recent study in which structures 5 and $\mathbf{6}$ (Figure 2) were used as transition state analogues in the preparation of imprinted polymers based on DVB as cross-linker

\section{Molecular dynamics simulations}

In order to gain better understanding of the differences and similarities of the interactions between template and functional monomer in the presence of different cross-linkers, molecular dynamics simulations were performed, modelling the pre-polymerization mixture over a timeframe of $20 \mathrm{~ns}$. The simulations were analysed in terms of densities of the MAA protons relative to the template molecules in order to identify which sites of the template are most relevant for interactions with the functional monomer (Figure 3). From the representations of the density grids it becomes obvious that there are two main interaction sites at the template for the formation of hydrogen bonds involving the MAA proton, namely the oxygen atom of the amide group and the doubly bound oxygen atom of the carbamate group. Significant differences between the spatial arrangements relative to the different diastereomers are, however, not observed. 


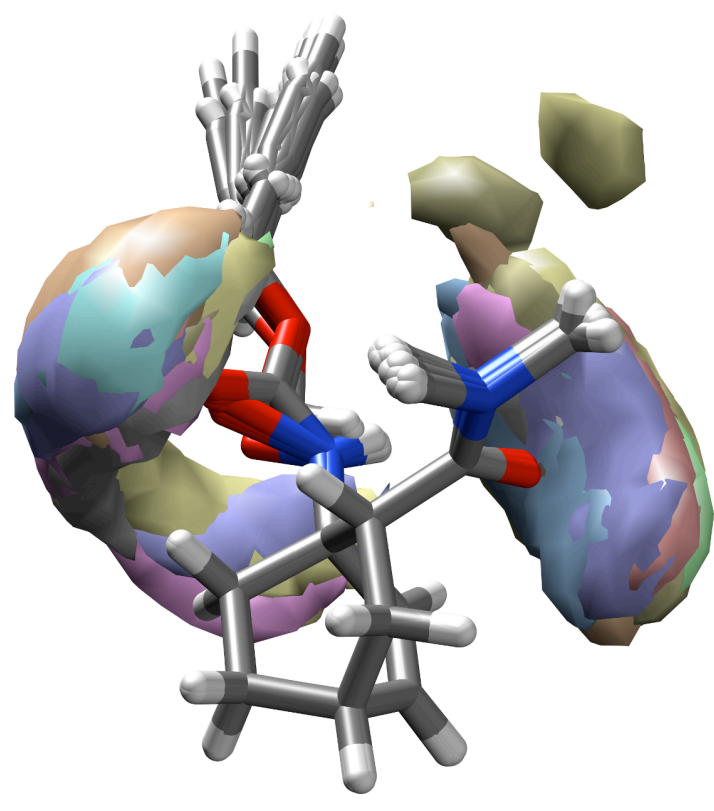

(a) endo-TSA, DVB as cross-linker

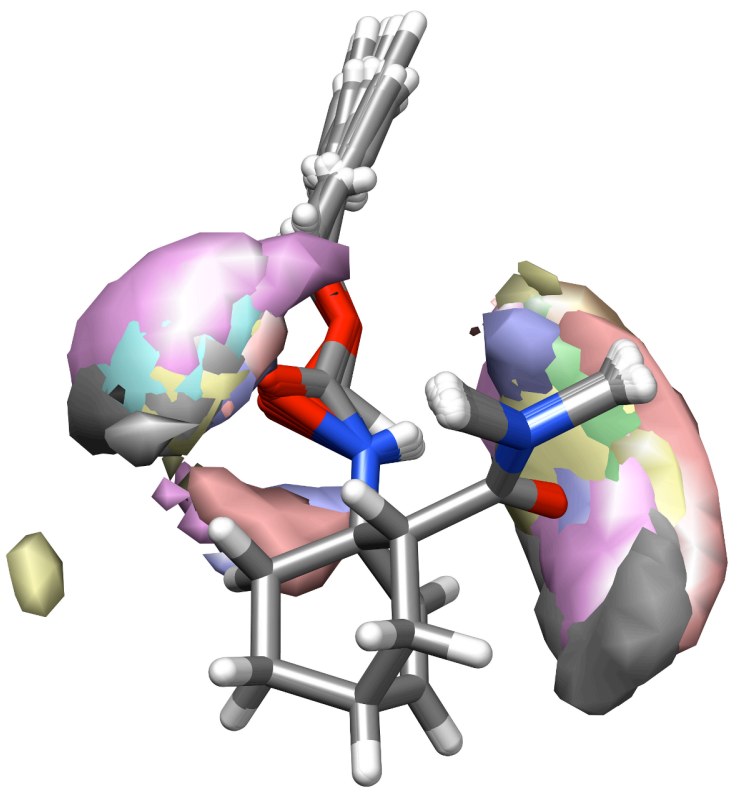

(c) endo-TSA, EGMA as cross-linker

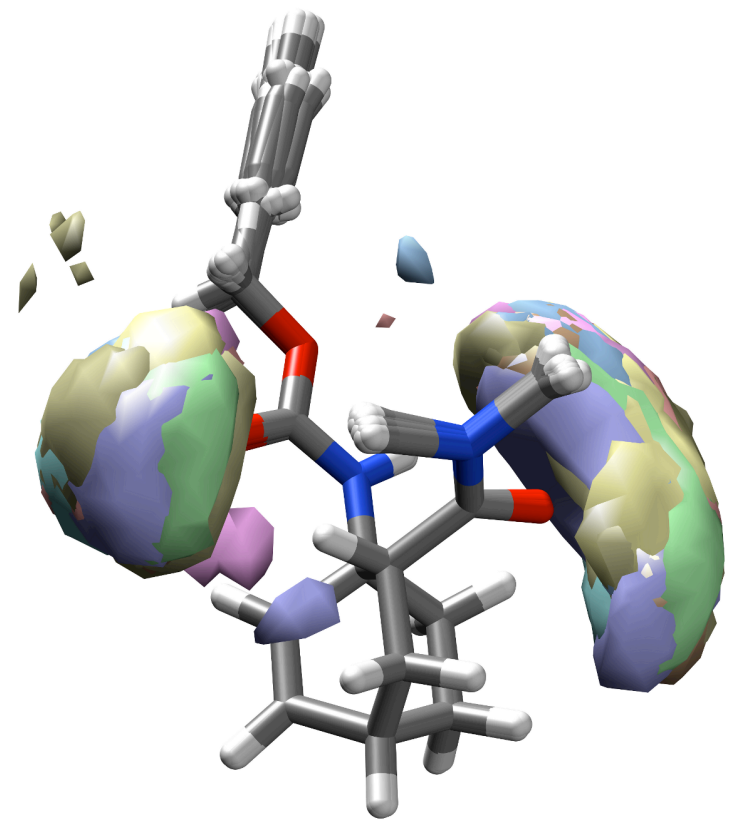

(b) exo-TSA, DVB as cross-linker

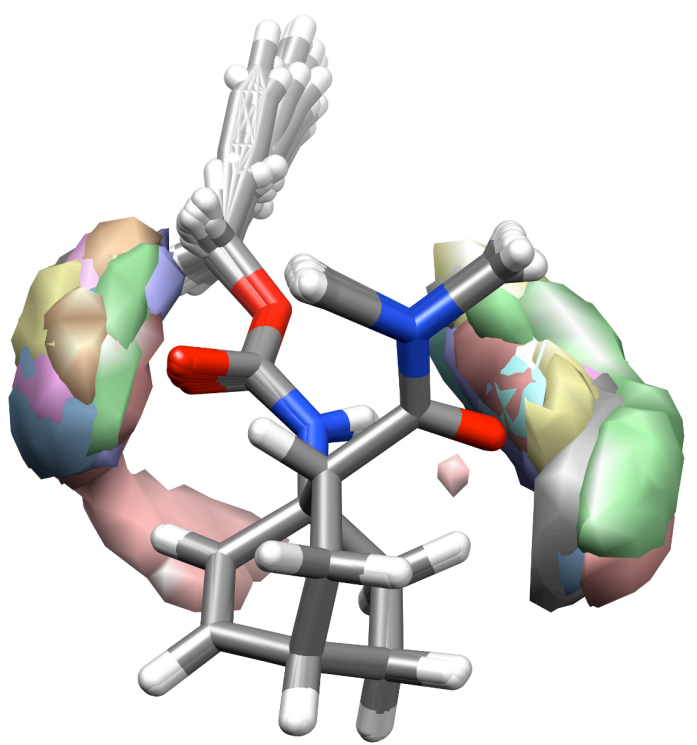

(d) exo-TSA, EGMA as cross-linker

Figure 3. Grid density analysis of the distribution of MAA protons relative to the time-averaged structures of the two different TSAs in the presence of the two different cross-linkers, respectively. Each colour represents the grid surface at $\mathbf{1 5}$ ps residence time for a single copy of the template.

These interactions, as well as possible interactions involving the carbamate proton, were studied more closely in terms of hydrogen bonds analysis with a $3.0 \AA$ distance and a $90^{\circ}$ angle cut-off for the interaction to count as a hydrogen bond. The results of this analysis are summarised in Table 1. 
Table 1. Occupancy and mean lifetime of hydrogen bonds in the systems modelled.

\begin{tabular}{|c|c|c|c|c|c|}
\hline \multirow{2}{*}{$\begin{array}{l}\text { Cross-linker } \\
\text { TSA }\end{array}$} & & \multicolumn{2}{|c|}{ EGMA } & \multicolumn{2}{|c|}{ DVB } \\
\hline & & endo & exo & endo & exo \\
\hline \multirow{2}{*}{$\mathrm{O}_{\text {amide }}-\mathrm{H}_{\mathrm{MAA}}$} & Occ. & $51.7 \%$ & $26.3 \%$ & $59.8 \%$ & $59.9 \%$ \\
\hline & $\bar{\tau}[\mathrm{ps}]$ & $5.4 \pm 3.5$ & $6.2 \pm 3.7$ & $4.9 \pm 3.6$ & $4.7 \pm 3.1$ \\
\hline \multirow{2}{*}{$\mathrm{O}_{\text {carbamate }}-\mathrm{H}_{\mathrm{MAA}}$} & Occ. & $13.6 \%$ & $12.7 \%$ & $22.5 \%$ & $25.5 \%$ \\
\hline & $\bar{\tau}[\mathrm{ps}]$ & $4.5 \pm 2.4$ & $4.8 \pm 2.5$ & $4.6 \pm 2.6$ & $4.5 \pm 2.7$ \\
\hline \multirow{2}{*}{$\mathrm{O}_{\text {MAA }}-\mathrm{H}_{\text {carbamate }}$} & Occ. & $20.7 \%$ & $8.1 \%$ & $24.0 \%$ & $22.0 \%$ \\
\hline & $\bar{\tau}[\mathrm{ps}]$ & $0.7 \pm 0.5$ & $0.7 \pm 0.5$ & $0.7 \pm 0.4$ & $0.8 \pm 0.4$ \\
\hline \multirow{2}{*}{$\mathrm{O}_{\mathrm{EGMA}}-\mathrm{H}_{\text {carbamate }}$} & Occ. & $6.5 \%$ & $15.0 \%$ & & \\
\hline & $\bar{\tau}[\mathrm{ps}]$ & $0.6 \pm 0.5$ & $0.6 \pm 0.5$ & & \\
\hline
\end{tabular}

One rather clear trend that can be found upon moving from DVB as cross-linker to EGMA is that the total relative occupation of the hydrogen bond to the carbamate oxygen atom is considerably lowered even though the strength of the bond (in terms of lifetime) does not change. For the hydrogen bond to the amide oxygen atom no such clear trend is to be found. In case of the endo-TSA the occupancy is only lowered slightly in the presence of EGMA as cross-linker as compared to the system with DVB as cross-linker. However, in case of the exo-TSA the occupancy is lowered by more than half. In both cases these changes are accompanied by similar relative changes in the occupancy of the hydrogen bond between the carbamate proton of the template and the doubly bond oxygen atom of MAA. This can safely be assumed to be due to the close proximity of the two groups in the structure of the TSA (cf. Figure 3), leading to a cooperative effect. This cooperative effect can also be the background to the rather large difference in occupancy of these two hydrogen bonds of the different TSA structures in the presence of EGMA. In case of the exo-TSA the much lower binding to MAA is contrasted by a higher occupancy of the hydrogen bond between the carbamate proton and one of the doubly bound oxygen atoms of EGMA. For these two occupancies the difference between the systems is similar (but differently signed), thus, the total binding of the carbamate proton is similar in all studied systems. In the systems with EGMA as cross-linker the proton binds in part to the EGMA, which - due to the cooperative effect - also lowers the binding of MAA protons to the amide oxygen atom. The difference in the relative distribution 
of the bonds of the carbamate proton is assumedly due to differences in the randomized starting geometries favouring one of the two similar binding modes over the other.

\section{Polymer synthesis and evaluation}

Imprinted polymers were prepared with a composition of $20 \mathrm{~mol} \%$ functional monomer (methacrylic acid, MAA) and $80 \mathrm{~mol} \%$ cross-linker (ethylene glycol dimethacrylate, EGMA) in the presence of $5 \mathrm{~mol} \%$ equivalents template (either endo- (5) or exo- (6) TSA). Thus, the polymers have the same molar composition as those used in our previous study. Physical and elemental characterisation of the obtained polymers is to be found in Table 2 .

Table 2: Elemental composition and physical analysis of polymers.

\begin{tabular}{lcccc}
\hline Polymer code & TSA & $\begin{array}{c}\text { Specific } \\
\text { swelling } \\
{\left[\mathrm{ml} \mathrm{g}^{-1}\right]}\end{array}$ & $\begin{array}{c}\text { Surface } \\
\text { area } \\
{\left[\mathrm{m}^{2} \mathrm{~g}^{-1}\right]}\end{array}$ & Anal. Calcd. [\%]* \\
\hline P(EGMA) ENDO & endo & 6.70 & 316.61 & C: $57.8, \mathrm{H}: 7.25, \mathrm{~N}:<0.3$ \\
P(EGMA) $)_{\text {EXO }}$ & exo & 7.01 & 277.11 & C: $57.55, \mathrm{H}: 7.3, \mathrm{~N}:<0.3$ \\
P(EGMA) $)_{\text {REF }}$ & - & 6.57 & 280.53 & C: $57.15, \mathrm{H}: 7.2, \mathrm{~N}:<0.3$ \\
\hline
\end{tabular}

* Theoretical calculations (based on complete incorporation initiator radicals): C: 60.51, H: 7.16, N: 0.28

After work-up of the polymers, batch binding studies with the endo- or exo-TSA at 0.1 and $1 \mathrm{mM}$, respectively, were carried out using the imprinted polymers and their controls in toluene. Table 3 shows the summary of the specific binding.

Table 3. Specific binding of the endo or exo TSA $(\mathrm{c}=1 \mathrm{mM}$ or $0.1 \mathrm{mM})$ in toluene to the different polymers. The uptakes are the specific uptakes, i.e. the binding to the control subtracted from the binding to the MIP.

\begin{tabular}{cccc}
\hline \multirow{2}{*}{ Ligand } & & \multicolumn{2}{c}{ Specific uptake [\%] } \\
\cline { 3 - 4 } & Polymer & $\mathbf{1 ~ m M}$ & $\mathbf{0 . 1} \mathbf{~ m M}$ \\
\hline endo TSA & $\mathrm{P}(\mathrm{EGMA})_{\mathrm{ENDO}}$ & $24.31 \pm 0.74$ & $38.60 \pm 2.24$ \\
endo TSA & $\mathrm{P}(\mathrm{EGMA})_{\mathrm{EXO}}$ & $21.65 \pm 1.91$ & $30.47 \pm 2.55$ \\
exo TSA & $\mathrm{P}(\mathrm{EGMA})_{\mathrm{ENDO}}$ & $19.51 \pm 2.55$ & $30.18 \pm 3.28$ \\
exo TSA & $\mathrm{P}(\mathrm{EGMA})_{\mathrm{EXO}}$ & $22.69 \pm 0.83$ & $31.35 \pm 7.87$ \\
\hline
\end{tabular}


The specific binding exhibited by the EGMA polymers is much higher than that of the corresponding DVB polymers (19-39\% as compared to $3-12 \%)$. While the larger surface area could lead to the expectation of higher non-specific binding, the higher specific binding shows that this effect is more than balanced by the formation of additional specific binding sites. Selectivity for the template utilized in its preparation could, however, only be observed for P(EGMA) $)_{\mathrm{ENDO}}$.

Assay

In order to investigate the catalytic properties of the polymers, i.e. $\mathrm{P}(\mathrm{EGMA})$, the reaction between diene (1) and $N, N$-dimethylacrylamide (2) in toluene was performed in the presence of each of the different polymers prepared. The formation of the products was monitored by HPLC.

When the reaction was carried out at room temperature the imprinted polymers increased the rate of formation of the endo-product approximately 6-fold, while the non-imprinted polymer gave a 4.5-fold increase as compared to the solvent reaction (Figure 4). As for the exoproduct, a significant increase in its formation could not be observed as concentrations remain below the detection limit. However, diastereoselectivity of the catalysis could not be observed, i.e. there is no observable difference in endo-product formation between the polymers imprinted with endo- and exo-TSAs. This, in turn, is in agreement with the results obtained from the batch binding studies where no significant diastereoselectivity of binding of the TSAs was observed. 


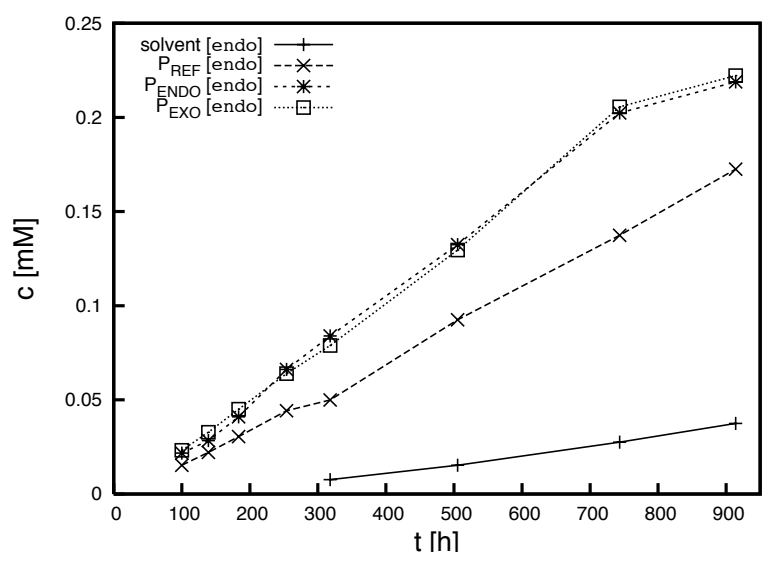

Figure 4. Formation of 3 in the presence of $P_{R E F}, P_{\text {ENDo, }}$ and $P_{\text {Exo }}$ and solvent only in toluene at room temperature.

In a second study the reaction was carried out at $120^{\circ} \mathrm{C}$. While formation of the endoproduct is initially $(\mathrm{t}<20 \mathrm{~h}$ ) slightly faster in presence of imprinted polymer as compared to both solvent reaction and control polymer, at longer reaction times its concentration is lower if polymer is present (Figure 5). In case of the exo-product the concentration in presence of polymer is at all sample times lower than for the solution reaction. Furthermore, the concentration of the exo-product is lower for the imprinted polymers than for the nonimprinted control polymer (the same phenomenon can be observed for the endo-product at reaction times $>60 \mathrm{~h}$, but there the trend is not conclusive). As for the room temperature study, stereoselectivity in terms of product formed in the presence of different polymers could not be observed.
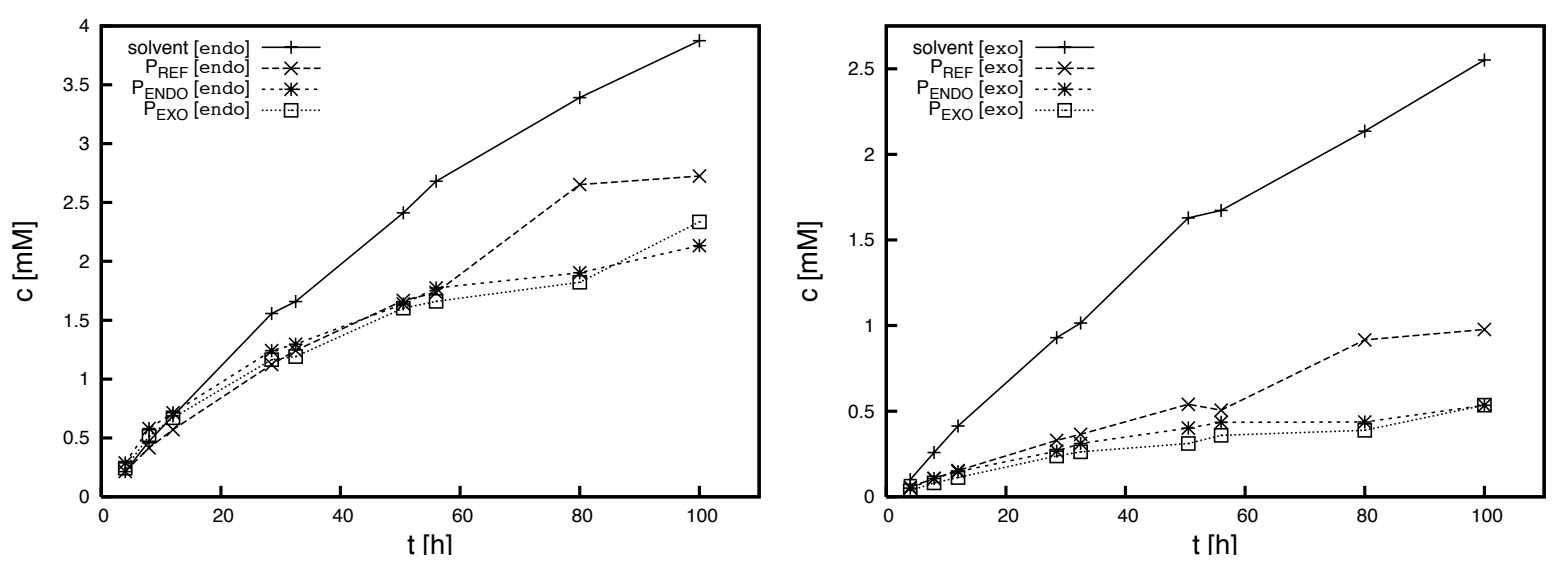

Figure 5. Formation of 3 and 4 in the presence of $P_{R E F}, P_{\text {ENDO, and }} P_{E X o}$ and solvent only in toluene at $120^{\circ} \mathrm{C}$. 
To further investigate the temperature dependence of the reaction, another series of experiments was conducted at $40{ }^{\circ} \mathrm{C}$. At this temperature the formation of the endo-product is up to 9.5 times faster in presence of polymer than in its absence (Figure 6). Remarkably, the difference between imprinted and non-imprinted polymers is far less distinguished in this study than at room temperature. This is especially true for the formation of exo-product, where there is (with respect to experimental error) no difference to be found between the different polymers' performance. Nonetheless, a 2-fold enhancement of reaction rate can be observed.
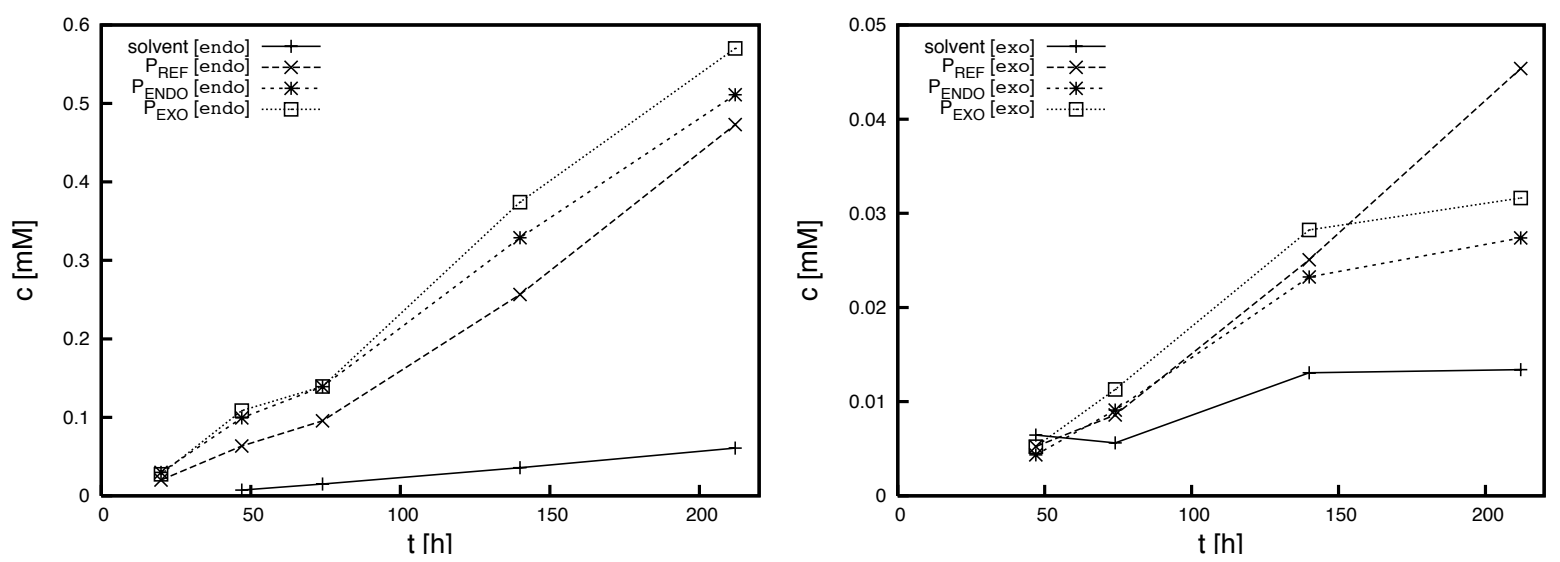

Figure 6. Formation of 3 and 4 in the presence of $P_{\text {REF }}$, $P_{E N D O}$, and $P_{E X o}$ and solvent only in toluene at $40{ }^{\circ} \mathrm{C}$.

Another reaction assay was performed at room temperature in presence of $20 \mathrm{mM}$ endo-TSA (5) as inhibitor. The result of this experiment was essentially the same as for the DVB-based polymers in our previously published study[35], i.e. the reaction rate was reduced by approximately $30 \%$ compared to the non-inhibited reaction.

\section{Discussion}

\section{Influence of cross-linker}

The first interesting finding in this study is the specific binding of template to the imprinted polymers, which is substantially higher for each of the EGMA polymer systems than for the comparable DVB-based polymers. This is especially to be highlighted as it is despite a larger surface area, which could be expected to lead to a larger extent of non-specific binding. 
Interestingly the higher specific binding observed in the batch binding studies does not lead to higher reaction rates. Even though a fundamental enhancement of the rate of product formation is observed at room temperature, the DVB-based polymers featured a more pronounced effect. Furthermore, the relative effect of inhibitor is similar for both systems, which implies similar ratios between the number of reactions taking place on the polymer surface and inside binding sites.

In the results of the molecular dynamics studies it can be seen that the interactions between MAA and the template are similar in the presence of both cross-linkers - the strength of the different interactions in terms of lifetime is essentially identical. In the case of EGMA as cross-linker, however, there is competition between the template and EGMA as acceptors of hydrogen bonds from MAA on one hand, and between MAA and EGMA as acceptors of hydrogen bonds from the TSA on the other hand. The larger diversity of interactions with the template in the system containing EGMA can easily explain the higher total binding observed as compared to the system containing DVB. Simultaneously, the higher specific binding observed for the EGMA system can be explained by the competitive binding of the different functionalities of functional monomer and cross-linker, leading to a situation in which functional groups that otherwise could lead to non-specific binding are involved in interactions between MAA and EGMA.

A common characteristic of the two different polymer systems is the lack of diastereoselectivity in terms of product formed in their presence. The fact that the choice of cross-linker does not have an impact on diastereoselectivity supports our previously made argument that the placement of a single double bond within the template structure is not sufficient to induce selective recognition. Nonetheless, the employment of EGMA-based 
polymers allowed for observation of exo-product formation in the presence of polymer. The formation over time parallels that of the endo-product - at a significantly lower rate.

\section{Influence of temperature}

Interestingly, the unprecedented temperature dependent behaviour of polymer activity, reported in our previous study[35], could be reproduced in spite of the fundamental change in the polymers' composition (the cross-linker makes up 80 mol\% of the reaction mixture). Even though the effect is less pronounced than for the DVB-based polymer, a considerable decline in reaction rate compared to the solution reaction could be observed at $120^{\circ} \mathrm{C}$.

However, due to the lesser extent of the effect, a more detailed analysis is possible. Amongst others, it can be observed that the rate of formation of the endo-product initially $(\mathrm{t}<20 \mathrm{~h})$ is slightly higher in the presence of imprinted polymers than in solvent or with the reference polymer. This indicates that the polymers, even at $120^{\circ} \mathrm{C}$, have some catalytic activity from the start which then, under the course of the experiment, is lost. This supports our theory that additional reactions of substrates and/or product of the Diels-Alder reaction with the polymer occur, blocking the specific binding sites and obstructing further reactions with its surface.

Another finding is that the activity of the imprinted polymers at longer reaction times becomes lower than that of the reference polymers. This could be explained by sites having high catalytic activity being also more reactive towards the inhibiting reaction, resulting in a stronger decrease for the imprinted polymers than for the non-imprinted.

A higher reactivity of the specific binding sites towards the side reaction is also supported by the data obtained at $40^{\circ} \mathrm{C}$. While the reaction rate is - as expected - much higher than at room temperature, the differences between the imprinted and reference polymers are much less distinct. This suggests a diminished effect of the specific binding sites, which in turn can be explained by the integrity of the binding sites being destroyed by side reactions, while on the polymer surface higher temperatures are required in order for this process to take place. 
Both the fact, that the unusual temperature dependent behaviour is more distinct for the polymer systems showing higher catalytic activity (i.e. the DVB-based polymers), and that the effect is more distinct for the imprinted than non-imprinted polymers, suggests that the process diminishing the polymers' catalytic activity is similar to the catalysed reaction itself, e.g. a reaction of substrates and/or product with residual double bonds in the polymer. 


\section{Conclusions}

In this study a series of molecularly imprinted polymers was designed with the help of analogues for two possible transition states of the Diels-Alder reaction of $\mathbf{1}$ and $\mathbf{2}$. The polymers show specific binding of the template employed in their synthesis and significant catalytic activity for the reaction studied.

Importantly, at high temperature $\left(120^{\circ} \mathrm{C}\right)$ significantly less product was formed in the presence of polymer than in its absence. This is analogous to a temperature-dependent behaviour that - by our group - only recently was reported for the first time for another imprinted polymer system. Furthermore, in this study we investigated the temperature dependence of the polymer's performance in more detail by performing the reaction assay at an additional temperature. The additional information obtained from this new polymer system not only supports our provisional theory that at higher temperature the polymer participates in the reaction, it also suggests that this reaction preferentially takes places within the catalytically active sites.

Finally, the equilibria in the pre-polymerization mixtures were studied by means of molecular dynamics simulations. The results illustrate the importance of different interactions of the template with functional monomer and cross-linker molecules, as well as interactions between functional monomer and cross-linker. Insights from these simulations were successfully applied to explain the different properties of the different polymer systems. This demonstrates once again the importance of the processes taking place in the pre-polymerization mixture for the imprinting process and ultimately for the performance of the resulting polymer. 


\section{Acknowledgements.}

We gratefully acknowledge the financial support of the Swedish Research Council (VR), the Swedish Knowledge Foundation (KKS), Graninge Foundation and the Linnæus University. We are indebted to Dr Sacha Legrand and Dr Jesper G. Wiklander (both Linnæus University) for the recording of 2D NMR spectra. 


\section{References}

[1] K.C. Nicolaou, S.A. Snyder, T. Montagnon, G. Vassilikogiannakis, Angew. Chem. Int. Ed. 41 (2002) 1668-1698.

[2] E.J. Corey, Angew. Chem. Int. Ed. 41 (2002) 1650-1667.

[3] U. Pindur, G. Lutz, C. Otto, Chem. Rev. 93 (1993) 741-761.

[4] M.R. Trembley, T.J. Dickerson, K.D. Janda, Adv. Synth. Catal. 343 (2001) 577-585.

[5] V.E. Gouverneur, K.N. Houk, B. de Pascual-Teresa, B. Beno, K.D. Janda, R.A. Lerner, Science. 262 (1993) 204-208.

[6] J.T. Yli-Kauhaluoma, J.A. Ashley, C.-H. Lo, L. Tucker, M.M. Wolfe, K.D. Janda, J. Am. Chem. Soc. 117 (1995) 7041-7047.

[7] C. Alexander, H.S. Andersson, L.I. Andersson, R.J. Ansell, N. Kirsch, I.A. Nicholls, J. O’Mahoney, M.J. Whitcombe, J. Mol. Recognit. 19 (2006) 106-180.

[8] D. Batra, K.J. Shea, Curr. Opin. Chem. Biol. 7 (2003) 434-442.

[9] K. Haupt, K. Mosbach, Chem. Rev. 100 (2000) 2495-2504.

[10] M. Komiyama, T. Takeuchi, T. Mukawa, H. Asanuma, Molecular Imprinting: From Fundamentals to Applications, Wiley-VCH, Weinheim, 2002.

[11] S. Piletsky, A. Turner [Eds.], Molecular Imprinting of Polymers, Landes Bioscience, Georgetown, 2006.

[12] B. Sellergren [Ed.], Molecularly Imprinted Polymers: Man-Made Mimics of Antibodies and Their Applications in Analytical Chemistry, Elsevier, Amsterdam, 2001.

[13] G. Wulff, Chem. Rev. 102 (2002) 1-28.

[14] M. Yan, O. Ramström [Eds.], Molecularly Imprinted Materials: Science and Technology, Dekker, New York, 2005.

[15] Y. Kawanami, T. Yunoki, A. Nakamura, K. Fujii, K. Umano, H. Yamauchi, K. Masuda, J. Mol. Catal. A 145 (1999) 107-110.

[16] J.M. Kim, K.D. Ahn, G. Wulff, Macromol. Chem. Phys. 202 (2001) 1105-1108.

[17] J.-Q. Liu, G. Wulff, J. Am. Chem. Soc. 130 (2008) 8044-8054.

[18] K. Ohkubo, Y. Urata, S. Hirota, Y. Funakoshi, S. Usui, K. Yoshinaga, J. Mol. Catal. A 101 (1995) L111-L114.

[19] K. Ohkubo, Y. Funakoshi, Y. Urata, S. Usui, T. Sagawa, J. Chem. Soc., Chem. Commun. (1995) 2143-2144.

[20] D.K. Robinson, K. Mosbach, J. Chem. Soc., Chem. Commun. (1989) 969-970. 
[21] T. Sagawa, H. Ihara, E.K. Mitamura, K. Ohkubo, Recent Res. Dev. Pure Appl. Chem. 3 (1999) 57-71.

[22] B. Sellergren, K.J. Shea, Tetrahedron: Asymmetry. 5 (1994) 1403-1406.

[23] B. Sellergren, R.N. Karmalkar, K.J. Shea, J. Org. Chem. 65 (2000) 4009-4027.

[24] A.G. Strikovsky, D. Kasper, M. Grün, B.S. Green, J. Hradil, G. Wulff, J. Am. Chem. Soc. 122 (2000) 6295-6296.

[25] G. Wulff, T. Gross, R. Schönfeld, Angew. Chem., Int. Ed. Engl. 36 (1997) 1962-1964.

[26] J. Svenson, N. Zheng, I.A. Nicholls, J. Am. Chem. Soc. 126 (2004) 8554-8560.

[27] J.V. Beach, K.J. Shea, J. Am. Chem. Soc. 116 (1994) 379-380.

[28] R. Müller, L.I. Andersson, K. Mosbach, Makromol. Chem., Rapid Commun. 14 (1993) 637-641.

[29] J. Hedin-Dahlström, J.P. Rosengren-Holmberg, S. Legrand, S. Wikman, I.A. Nicholls, J. Org. Chem. 71 (2006) 4845-4853.

[30] J. Matsui, I.A. Nicholls, I. Karube, K. Mosbach, J. Org. Chem. 61 (1996) 5414-5417.

[31] A.N. Cammidge, N.J. Baines, R.K. Bellingham, Chem. Commun. (2001) 2588-2589.

[32] X.-C. Li, K. Mosbach, Macromol. Rapid Commun. 18 (1997) 609-615.

[33] B.P. Santora, A.O. Larsen, M.R. Gagné, Organometallics. 17 (1998) 3138-3140.

[34] A. Viesnjevsky, R. Schomäcker, E. Yilmaz, O. Brüggemann, Catal. Commun. 6 (2005) 601-606.

[35] N. Kirsch, J. Hedin-Dahlström, H. Henschel, M.J. Whitcombe, S. Wikman, I.A. Nicholls, J. Mol. Catal. B: Enzym. 58 (2009) 110-117.

[36] J.G. Karlsson, L.I. Andersson, I.A. Nicholls, Anal. Chim. Acta. 435 (2001) 57-64.

[37] D.A. Case, T.A. Darden, T.E. Cheatham, III, C.L. Simmerling, J. Wang, R.E. Duke, R. Luo, M. Crowley, R.C. Walker, W. Zhang, K.M. Merz, B. Wang, S. Hayik, A. Roitberg, G. Seabra, I. Kolossváry, K.F. Wong, F. Paesani, J. Vanicek, X. Wu, S.R. Brozell, T. Steinbrecher, H. Gohlke, L. Yang, C. Tan, J. Mongan, V. Hornak, G. Cui, D.H. Mathews, M.G. Seetin, C. Sagui, V. Babin, and P.A. Kollman (2008), AMBER 10, University of California, San Francisco.

[38] B.C.G. Karlsson, J. O’Mahony, J.G. Karlsson, H. Bengtsson, L.A. Eriksson, I.A. Nicholls, J. Am. Chem. Soc. 131 (2009) 13297-13304. 